\title{
Limit Cycle Control Using Energy Function Regulation with Friction Compensation
}

\author{
Gianluca Garofalo and Christian Ott
}

\begin{abstract}
In this paper we consider the problem of generating periodic solutions for fully actuated robots with unknown disturbances, which can be modeled using a regressor matrix. We extend our previous work on limit cycle control based on energy function regulation for the case when disturbance torques are acting on the system, e.g. torques due to friction. Since the controller is designed in two independent steps, the compensation of the friction cannot be carried on with standard techniques and it will be split in two steps as well. In the first one we reduce the dimension of the dynamical system and use a sliding mode approach for friction compensation, in the second we produce the desired limit cycle and use an adaptive approach for friction compensation. Crucial for the analysis is the concept of conditional attractiveness with semidefinite Lyapunov functions, that we formulate in this paper to show the attractiveness of the closed orbit of the whole system, even if it is designed assuming a reduced dynamics. Finally, we validate our approach with experiments on a humanoid robot.
\end{abstract}

\section{INTRODUCTION}

Periodic tasks are of interests in quite a lot of different applications, like running and walking [1]-[3]. In lots of these cases it is more important to stay on a prescribed orbit in the state space, rather than following the exact position in time along the desired curve. For these applications tracking a trajectory might not be the best solution, as already addressed in [4]. Inspired by the need of controlling the energy to achieve a periodic behavior as shown in [3], here we propose a feedback control law to produce closed attractive orbits for fully actuated mechanical multibody systems with unknown disturbances, which can be modeled using a regressors matrix, e.g. torques due to friction. We create an attractive path in the state space for the closed loop system, extending our previous results where the ideal frictionless case was considered [5]. Although the basic idea is the same as in our previous work, where a simple PD action was used, here to cope with the presence of the disturbance we utilize sliding mode and adaptive techniques at the same time.

The problem of orbital stabilization has been already addressed in other works, like [6]-[8]. In [6], [7] the authors extend the potential field controller adding powercontinuous terms, while in the second the concepts of virtual constraint and feedback linearization are used to obtain a closed loop system that generates its own stable periodic motion. In our approach we formulate the problem based on the nullspace decomposition introduced in [9]-[11]. In this way, we take advantage of the passivity property of the

The authors are with the Institute of Robotics and Mechatronics, German Aerospace Center (DLR), Wessling, Germany. gianluca.garofalo(at)dlr.de

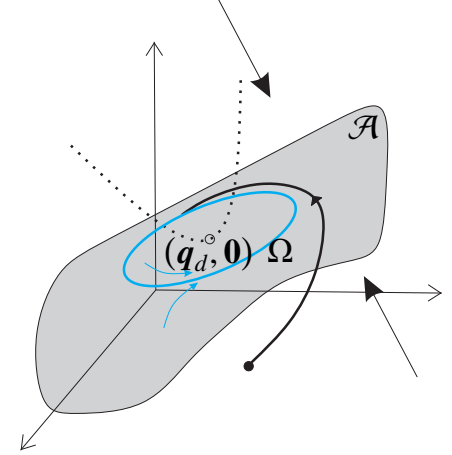

Fig. 1: Conceptual illustration of the control action. The system is forced to reach a subset of the state space, where we produce an attractive closed orbit.

system and do not completely alter the original dynamics of the system through feedback linearization. Secondly, we completely separate the problem of producing the limit cycle from the virtual constraints, instead of modifying the latter for achieving the first. The input torques are split in the ones necessary for producing the limit cycle and the ones necessary for satisfying the virtual constraints. These, in turn, are responsible for the configuration in which the system will oscillate. Finally, relating the input to the energy to be injected in the system could reduce the control effort in presence of additional sources (e.g. energy stored in elastic actuators). This is particular important since the focus of our research is to find new and efficient methods to produce periodic motions that can be used in general and in particular for locomotion [3], [5], [12]-[15]. Therefore, instead of forcing the system to follow a close integral curve of a vector field via passive control action as in [6], [7], we aim at achieving a stable limit cycle in the state space by regulating a virtual energy function on a 1 - dimensional submanifold of the configuration space, as it is conceptually sketched in Fig. 1. This virtual energy function consists of the physical kinetic energy and a virtual potential energy, which represents an additional design element in the controller. Intuitively, the freedom in choosing this potential can be used to achieve energy efficient motion in mechanical systems with compliant actuation. Nevertheless, it should be also mentioned that in [8] the more complicated problem of controlling an underactuated system is considered, which here we do not take into account yet.

On the other hand, the problem of coping with uncertain models has been widely studied in robotics both from a 
robust control point of view [16]-[19] and from an adaptive control point of view [20]-[22]. A complete review of these approaches is beyond the scope of the paper and we will simply point out that, since the design of the controller is obtained in two independent steps, the compensation of the friction cannot be carried out with standard techniques. As for the control design, also the friction will be taken into account in two different steps. In the first one, we use a sliding mode approach to be robust against the unknown disturbances, while in the second we use an adaptive approach for friction compensation. Crucial for the analysis is the concept of conditional attractiveness with semidefinite Lyapunov functions, that we formulate in this paper to show the attractiveness of the closed orbit of the whole system, even if it is designed assuming a reduced dynamics.

The paper is organized as follows. In Section II we present the model and the assumptions used in the paper, together with a coordinate transformation used to separate the enforcing of the constraints from the limit cycle generation. Section III is the main contribution of the paper. There we explain the extension of our previous work in order to cope with model uncertainties like friction. Experimental results will be presented in Section IV, where the humanoid robot TORO is used to validate the proposed approach. The paper is concluded with a final discussion and outline of future work in Section V.

\section{Model AND PRELIMINARIES}

We consider the class of fully actuated, mechanical systems which can be modeled by the Euler - Lagrange principle. The result is a nonlinear system modeled by a set of highly coupled nonlinear differential equations. Assuming to have $n-\mathrm{DoF}$, the dynamic model is:

$$
\boldsymbol{M}(\boldsymbol{q}) \ddot{\boldsymbol{q}}+\boldsymbol{C}(\boldsymbol{q}, \dot{\boldsymbol{q}}) \dot{\boldsymbol{q}}+\boldsymbol{g}(\boldsymbol{q})=\boldsymbol{\tau}+\tau_{f},
$$

where $\boldsymbol{q}, \dot{\boldsymbol{q}} \in \mathbb{R}^{n}$ constitute together the state of the system, being $\boldsymbol{q}$ the joint position and $\dot{\boldsymbol{q}}$ the joint velocity. The input to the system is the torque $\tau \in \mathbb{R}^{n}$ provided by the motors. Finally, $\boldsymbol{M}(\boldsymbol{q}) \in \mathbb{R}^{n \times n}$ is the symmetric positive definite inertia matrix, $\boldsymbol{C}(\boldsymbol{q}, \dot{\boldsymbol{q}}) \in \mathbb{R}^{n \times n}$ is the Coriolis matrix, $\boldsymbol{g}(\boldsymbol{q})=\nabla_{\boldsymbol{q}} U_{g}(\boldsymbol{q}) \in \mathbb{R}^{n}$ is the gravity torque vector ${ }^{1}$ and $U_{g}(\boldsymbol{q})$ the gravitational potential. Additionally, we assume that the unknown torques $\boldsymbol{\tau}_{f} \in \mathbb{R}^{n}$ are acting as a disturbance.

Assumptions: The following properties are assumed

$$
\begin{array}{ll}
\text { - } & \boldsymbol{\tau}_{f}=\boldsymbol{Y}(\boldsymbol{q}, \dot{\boldsymbol{q}}) \boldsymbol{\pi} \\
\text { - } & \left\|\boldsymbol{\tau}_{f}\right\| \leq \beta_{1}\|\boldsymbol{q}\|+\beta_{2}\|\dot{\boldsymbol{q}}\|
\end{array}
$$

where $\beta_{1}, \beta_{2} \in \mathbb{R}$ are positive scalars, $\pi \in \mathbb{R}^{p}$ is a constant vector of unknown parameters and $\boldsymbol{Y}(\boldsymbol{q}, \dot{\boldsymbol{q}}) \in \mathbb{R}^{n \times p}$ is the so called regressor matrix, whose entries, on the contrary, are assumed to be known.

Viscous and Coulomb friction, as well as constant torques and Cartesian forces, are classical examples of disturbances that can be modeled in this way.

\footnotetext{
${ }^{1}$ With the symbol $\nabla_{x}(\cdot)$ we are indicating $\left(\frac{\partial(\cdot)}{\partial x}\right)^{T}$ in order to ease the notation.
}

\section{A. Coordinate transformation}

The submanifold on which we want to force the system to evolve is defined by means of a configuration dependent function $\boldsymbol{x}=\boldsymbol{x}(\boldsymbol{q})$. Given the function $\boldsymbol{x}=\boldsymbol{x}(\boldsymbol{q})$, we assume that the Jacobian matrix $\boldsymbol{J}(\boldsymbol{q}) \in \mathbb{R}^{(n-1) \times n}$ of the mapping $\boldsymbol{x}: \mathbb{R}^{n} \rightarrow \mathbb{R}^{n-1}$ is a full rank matrix. In this case, $\boldsymbol{x}(\boldsymbol{q})=\mathbf{0}$ defines a 1 - dimensional submanifold of the configuration space [23]. Accordingly, we can write the dynamics of the system with a new set of coordinates, as in [9]-[11]. We first compute a nullspace base matrix ${ }^{2} \boldsymbol{Z}(\boldsymbol{q}) \in \mathbb{R}^{1 \times n}$ which allows us to obtain the directions orthogonal to the submanifold, then we use $\boldsymbol{Z}(\boldsymbol{q})$ to compute a dynamically consistent ${ }^{3}$ nullspace projector $\boldsymbol{N}(\boldsymbol{q}) \in \mathbb{R}^{1 \times n}$, which will be part of the extended Jacobian matrix $\boldsymbol{J}_{N}(\boldsymbol{q}) \in \mathbb{R}^{n \times n}$, such that

$$
\left[\begin{array}{c}
\dot{\boldsymbol{x}} \\
v
\end{array}\right]=\boldsymbol{J}_{N}(\boldsymbol{q}) \dot{\boldsymbol{q}}=\left[\begin{array}{c}
\boldsymbol{J}(\boldsymbol{q}) \\
N(\boldsymbol{q})
\end{array}\right] \dot{\boldsymbol{q}}
$$

where $\boldsymbol{N}(\boldsymbol{q})=\left(\boldsymbol{Z}(\boldsymbol{q}) \boldsymbol{M}(\boldsymbol{q}) \boldsymbol{Z}^{T}(\boldsymbol{q})\right)^{-1} \boldsymbol{Z}(\boldsymbol{q}) \boldsymbol{M}(\boldsymbol{q})$ and $v$ is an additional nullspace velocity. One can show that by this choice the extended Jacobian $\boldsymbol{J}_{N}(\boldsymbol{q})$ is non singular and the inverse is given by

$$
\boldsymbol{J}_{N}^{-1}(\boldsymbol{q})=\left[\begin{array}{ll}
\boldsymbol{J}^{+_{M}}(\boldsymbol{q}) & \boldsymbol{Z}^{T}(\boldsymbol{q})
\end{array}\right],
$$

where $\boldsymbol{J}^{+_{M}}(\boldsymbol{q})$ denotes the dynamically consistent weighted pseudo inverse defined as

$$
\boldsymbol{J}^{+M}(\boldsymbol{q})=\boldsymbol{M}^{-1}(\boldsymbol{q}) \boldsymbol{J}^{T}(\boldsymbol{q})\left(\boldsymbol{J}(\boldsymbol{q}) \boldsymbol{M}^{-1}(\boldsymbol{q}) \boldsymbol{J}^{T}(\boldsymbol{q})\right)^{-1} .
$$

The joint velocity can thus be computed from the Cartesian velocity and the nullspace velocity via

$$
\dot{\boldsymbol{q}}=\boldsymbol{J}^{+_{M}}(\boldsymbol{q}) \dot{\boldsymbol{x}}+\boldsymbol{Z}^{T}(\boldsymbol{q}) v .
$$

From (2) and (5) it is straightforward to rewrite (1) in the extended velocity coordinates as

$$
\boldsymbol{\Lambda}(\boldsymbol{q})\left[\begin{array}{c}
\ddot{x} \\
\dot{v}
\end{array}\right]+\boldsymbol{\Gamma}(\boldsymbol{q}, \dot{\boldsymbol{q}})\left[\begin{array}{c}
\dot{\boldsymbol{x}} \\
v
\end{array}\right]=\boldsymbol{J}_{N}^{-T}(\boldsymbol{q})\left(\tau+\boldsymbol{\tau}_{f}-\boldsymbol{g}(\boldsymbol{q})\right),
$$

with the matrices $\boldsymbol{\Lambda}(\boldsymbol{q})$ and $\boldsymbol{\Gamma}(\boldsymbol{q}, \dot{\boldsymbol{q}})$ given by ${ }^{4}$

$$
\begin{aligned}
& \boldsymbol{\Lambda}(\boldsymbol{q})=\left[\begin{array}{cc}
\boldsymbol{\Lambda}_{x}(\boldsymbol{q}) & \mathbf{0} \\
\mathbf{0} & \Lambda_{n}(\boldsymbol{q})
\end{array}\right] \\
& \boldsymbol{\Lambda}_{x}(\boldsymbol{q})=\left(\boldsymbol{J}(\boldsymbol{q}) \boldsymbol{M}^{-1}(\boldsymbol{q}) \boldsymbol{J}^{T}(\boldsymbol{q})\right)^{-1} \quad \Lambda_{n}(\boldsymbol{q})=\boldsymbol{Z}(\boldsymbol{q}) \boldsymbol{M}(\boldsymbol{q}) \boldsymbol{Z}^{T}(\boldsymbol{q})
\end{aligned}
$$

and (omitting the dependences)

$$
\begin{aligned}
& \boldsymbol{\Gamma}(\boldsymbol{q}, \dot{\boldsymbol{q}})=\left[\begin{array}{cc}
\boldsymbol{\Gamma}_{x}(\boldsymbol{q}, \dot{\boldsymbol{q}}) & \boldsymbol{\Gamma}_{x n}(\boldsymbol{q}, \dot{\boldsymbol{q}}) \\
\boldsymbol{\Gamma}_{n x}(\boldsymbol{q}, \dot{\boldsymbol{q}}) & \Gamma_{n}(\boldsymbol{q}, \dot{\boldsymbol{q}})
\end{array}\right] \\
& \boldsymbol{\Gamma}_{x}=\boldsymbol{\Lambda}_{x}\left(\boldsymbol{J} \boldsymbol{M}^{-1} \boldsymbol{C}-\dot{\boldsymbol{J}}\right) \boldsymbol{J}^{+m} \\
& \boldsymbol{\Gamma}_{n x}=-\boldsymbol{\Gamma}_{x n}^{T} \\
& \boldsymbol{\Gamma}_{x n}=\boldsymbol{\Lambda}_{x}\left(\boldsymbol{J} \boldsymbol{M}^{-1} \boldsymbol{C}-\dot{\boldsymbol{J}}\right) \boldsymbol{Z}^{T} \\
& { }^{2} \text { I.e. it fulfills the condition } \boldsymbol{J}(\boldsymbol{q}) \boldsymbol{Z}^{T}(\boldsymbol{q})=\mathbf{0} . \\
& { }^{3} \text { I.e. it fulfills the condition } \boldsymbol{J}(\boldsymbol{q}) \boldsymbol{M}^{-1}(\boldsymbol{q}) \boldsymbol{N}^{T}(\boldsymbol{q})=\mathbf{0} \text {. } \\
& { }^{4} \text { Notice that using a dynamically consistent nullspace projector the } \\
& \text { matrix } \boldsymbol{\Lambda}(\boldsymbol{q}) \text { is block diagonal. }
\end{aligned}
$$




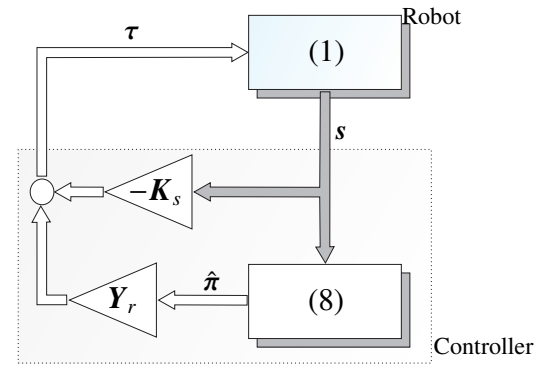

Fig. 2: The adaptive Slotine - Li control. The matrix $\boldsymbol{K}_{s}$ is positive definite and diagonal.

\section{Controller DESIGN}

In this section we present the control law that extends our previous results on generation of asymptotically stable limit cycles [5]. As in [5], the idea is to impose $n-1$ constraints to the system and generate a limit cycle for the zero dynamics. Although, the proposed controller is able to deal with model uncertainty of the type presented in Section II, e.g. disturbances due to friction. Since the design of the controller is carried on in two independent steps, the friction compensation cannot be achieved with standard techniques, as we will discuss in more details later on in Section III-D. To this end, the part of the controller responsible for forcing the system to evolve on a 1 - dimensional submanifold of the configuration space is modified using a sliding mode approach as in [19], which is robust to model uncertainty and guarantees asymptotic convergence. For the "remaining dynamics", on the other hand, it is not as straightforward to apply a sliding mode approach. For this reason, in the nullspace we use an adaptive approach to compensate for the disturbance as in [20].

Before presenting the main result of the paper, the previous methods are quickly reviewed in order to make the reader familiar with the terminology and the notation.

\section{A. The adaptive control in a nutshell}

The adaptive tracking control law presented in [20] is given by a feedback term proportional to the sliding variable $\boldsymbol{s}$ (which is a linear combination of the velocity and position tracking errors) and a feedforward term which uses an estimation of the unknown parameters. Given a positive definite matrix $\boldsymbol{P}$, the estimation is computed using an adaptive law which renders the positive definite function

$$
V_{\pi}=\frac{1}{2}\left(\boldsymbol{s}^{T} \boldsymbol{M}(\boldsymbol{q}) \boldsymbol{s}+\tilde{\pi}^{T} \boldsymbol{P}^{-1} \tilde{\boldsymbol{\pi}}\right),
$$

a Lyapunov function, where $\tilde{\pi}=\hat{\pi}-\pi$ is the error between the estimated and real parameters. In particular, the estimation is updated as

$$
\dot{\hat{\boldsymbol{\pi}}}=-\boldsymbol{P} \boldsymbol{Y}_{r}^{T}\left(\boldsymbol{q}, \dot{\boldsymbol{q}}, \dot{\boldsymbol{q}}_{r}, \ddot{\boldsymbol{q}}_{r}\right) \boldsymbol{s}
$$

where $\boldsymbol{Y}_{r}$ is the Slotine - Li regressor and $\dot{\boldsymbol{q}}_{r}=\dot{\boldsymbol{q}}-\boldsymbol{s}$. The control scheme is as in Fig. 2.

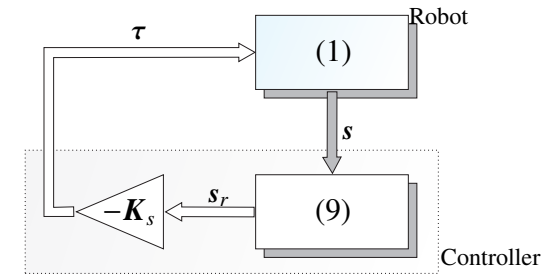

Fig. 3: Dynamic sliding PID control. The matrix $\boldsymbol{K}_{s}$ is positive definite and diagonal.

\section{B. The dynamic sliding PID control in a nutshell}

The dynamic sliding PID control [19] uses, as the control law in [20], a sliding variable $s$. Nevertheless, unlike in [20], the control input is not chosen in order to obtain an asymptotically stable dynamics for the sliding variable, but to obtain a stable dynamics in terms of the output $s_{r}$ of the dynamic system

$$
\begin{aligned}
\dot{\boldsymbol{s}}_{d} & =-\kappa \boldsymbol{s}_{d} \\
\dot{\boldsymbol{\sigma}} & =\operatorname{sgn}(\tilde{\boldsymbol{s}}) \\
\boldsymbol{s}_{r} & =\boldsymbol{Q}_{s} \boldsymbol{\sigma}+\tilde{\boldsymbol{s}},
\end{aligned}
$$

with $\tilde{\boldsymbol{s}}=\boldsymbol{s}-\boldsymbol{s}_{d}$, being $\boldsymbol{s}$ the input and $\boldsymbol{s}_{d}, \boldsymbol{\sigma}$ the state. The latter is initialized as $\boldsymbol{s}_{d}\left(t_{0}\right)=\boldsymbol{s}\left(t_{0}\right)$, i.e. the value of $\boldsymbol{s}$ at the initial time $t_{0}$, and $\boldsymbol{\sigma}\left(t_{0}\right)=\mathbf{0}$. Finally, $\kappa>0, \boldsymbol{Q}_{s}$ is a positive definite diagonal matrix and $\operatorname{sgn}(\boldsymbol{a})=\left[\operatorname{sgn}\left(a_{1}\right) \ldots \operatorname{sgn}\left(a_{n}\right)\right]^{T}$, for $\boldsymbol{a} \in \mathbb{R}^{n}$. Using a control input proportional to $\boldsymbol{s}_{r}$, as it is shown in Fig. 3, and given some bounds for the terms present in the dynamic model of the robot, it is possible to prove the boundedness of $\boldsymbol{s}_{r}$ and $\dot{\boldsymbol{s}}_{r}$. The latter allows to conclude that a sliding mode is established for all time on the error variable $\tilde{\boldsymbol{s}}$. To this end, the following second order dynamical system defined by (9)

$$
\dot{\tilde{\boldsymbol{s}}}=-\boldsymbol{Q}_{s} \operatorname{sgn}(\tilde{\boldsymbol{s}})+\dot{\boldsymbol{s}}_{r},
$$

together with the Lyapunov function

$$
V_{s}=\frac{1}{2} \tilde{\boldsymbol{s}}^{T} \tilde{\boldsymbol{s}}
$$

are used, since they imply the sliding mode condition $\tilde{\boldsymbol{s}}^{T} \dot{\tilde{\boldsymbol{S}}} \leq$ $-\mu|\tilde{\boldsymbol{s}}|, \mu>0$ [24]. In this way, the tracking errors are constrained to evolve on a submanifold that has an exponential solution toward zero [19].

\section{Main result}

For a given desired configuration $\boldsymbol{q}_{d} \in \mathbb{R}^{n}$, such that $\boldsymbol{x}\left(\boldsymbol{q}_{d}\right)=\mathbf{0}$, let $U(\boldsymbol{q})$ be a positive definite $C^{1}$ function on the 1 - dimensional submanifold defined by $\boldsymbol{x}(\boldsymbol{q})=\mathbf{0}$ and having its minimum at $\boldsymbol{q}_{d}$. In other words, we require that $\boldsymbol{q}_{d}, U(\boldsymbol{q})$ and $\boldsymbol{x}(\boldsymbol{q})$ are chosen such that $U(\boldsymbol{q})$ has a unique constrained local minimum at $\boldsymbol{q}_{d}$, i.e.

- $U(\boldsymbol{q}) \geq 0$ and $U(\boldsymbol{q})=0 \Longleftrightarrow \boldsymbol{q}=\boldsymbol{q}_{d}, \forall \boldsymbol{q}: \boldsymbol{x}(\boldsymbol{q})=\mathbf{0}$

- $\boldsymbol{x}\left(\boldsymbol{q}_{d}\right)=\mathbf{0}$. 
Consequently, the desired configuration $\boldsymbol{q}_{d}$ can be obtained as

$$
\begin{array}{r}
\boldsymbol{q}_{d}=\arg \min _{\boldsymbol{q}} U(\boldsymbol{q}) \\
\text { s.t. } \boldsymbol{x}(\boldsymbol{q})=\mathbf{0} .
\end{array}
$$

The necessary condition for optimality of the minimization problem, obtained using the Lagrange multipliers $\lambda$, is

$$
\nabla_{\boldsymbol{q}} U(\boldsymbol{q})+\boldsymbol{J}^{T}(\boldsymbol{q}) \boldsymbol{\lambda}=0,
$$

so that pre-multiplying by the nullspace base matrix $\boldsymbol{Z}(\boldsymbol{q})$ we obtain $n(\boldsymbol{q}):=\boldsymbol{Z}(\boldsymbol{q}) \nabla_{\boldsymbol{q}} U(\boldsymbol{q})=0$, where $n(\boldsymbol{q})$ can be seen as a local nullspace coordinate [25]. Concluding, $\boldsymbol{q}_{d}$ is the unique configuration with coordinates $\boldsymbol{x}\left(\boldsymbol{q}_{d}\right)=\mathbf{0}, n\left(\boldsymbol{q}_{d}\right)=0$.

At this point, the function $U(\boldsymbol{q})$ is used to define the "energy - like" function

$$
H(\boldsymbol{q}, \dot{\boldsymbol{q}})=\frac{1}{2} \dot{\boldsymbol{q}}^{T} \boldsymbol{M}(\boldsymbol{q}) \dot{\boldsymbol{q}}+U(\boldsymbol{q}),
$$

consisting of the physical kinetic energy and a virtual potential energy. Defining $\tilde{H}=H(\boldsymbol{q}, \dot{\boldsymbol{q}})-H_{d}$, our goal is to regulate $H(\boldsymbol{q}, \dot{\boldsymbol{q}})$ to the desired value $H_{d}$, i.e. $\tilde{H} \rightarrow 0$ as $t \rightarrow \infty$. The desired value $H_{d}$ influences the shape of the limit cycle. As rule of thumb, one can consider that when the regulation goal is achieved and the kinetic energy is zero, the desired energy is equal to the virtual potential energy and therefore it directly influences the maximum amplitude of the oscillations.

According to the dynamic sliding PID control, we define the sliding variable as

$$
s=\dot{x}+D_{s} x,
$$

and the nominal reference as

$$
\dot{\boldsymbol{x}}_{r}=\dot{\boldsymbol{x}}-\boldsymbol{s}_{r}=-\boldsymbol{D}_{s} \boldsymbol{x}+\boldsymbol{s}_{d}-\boldsymbol{Q}_{s} \boldsymbol{\sigma},
$$

being $\boldsymbol{s}_{r}$ the output of the system (9). Given the system (1) with Assumption (A1) - (A2), let $K_{H}, \alpha, \kappa \in \mathbb{R}$ be positive scalars, $\boldsymbol{D}_{s}, \boldsymbol{K}_{s}, \boldsymbol{Q}_{s} \in \mathbb{R}^{(n-1) \times(n-1)}$ positive definite diagonal matrices and $\boldsymbol{P} \in \mathbb{R}^{p \times p}$ a symmetric positive definite matrix, then the proposed nonlinear controller is (omitting the dependences)

$$
\boldsymbol{\tau}=\boldsymbol{g}+\boldsymbol{J}_{N}^{T}\left(\left[\begin{array}{cc}
\mathbf{0} & \boldsymbol{\Gamma}_{x n} \\
-\boldsymbol{\Gamma}_{x n}^{T} & \mathbf{0}
\end{array}\right]\left[\begin{array}{c}
\dot{x} \\
v
\end{array}\right]+\left[\begin{array}{c}
\boldsymbol{f}_{x} \\
f_{n}
\end{array}\right]\right)
$$

where

$$
\begin{aligned}
\boldsymbol{f}_{x} & =\boldsymbol{\Lambda}_{x} \ddot{\boldsymbol{x}}_{r}+\boldsymbol{\Gamma}_{x} \dot{\boldsymbol{x}}_{r}-\boldsymbol{K}_{s} \boldsymbol{s}_{r} \\
f_{n} & =-K_{H} \tilde{H} v+\boldsymbol{Z}\left(\boldsymbol{Y} \hat{\boldsymbol{\pi}}-\nabla_{\boldsymbol{q}} U\right) .
\end{aligned}
$$

Using the state vector $\chi=\left(\boldsymbol{q}, \dot{\boldsymbol{x}}, v, \boldsymbol{s}_{d}, \boldsymbol{\sigma}, \hat{\boldsymbol{\pi}}\right)$, the closed loop system is:

$$
\begin{aligned}
& \dot{\boldsymbol{q}}=\boldsymbol{J}^{+{ }_{M}} \dot{\boldsymbol{x}}+\boldsymbol{Z}^{T} v \\
& \boldsymbol{\Lambda}_{x} \dot{\boldsymbol{s}}_{r}+\left(\boldsymbol{\Gamma}_{x}+\boldsymbol{K}_{s}\right) \boldsymbol{s}_{r}=\boldsymbol{J}^{{ }_{M} T} \boldsymbol{Y} \boldsymbol{\pi} \\
& \Lambda_{n} \dot{v}+\left(\Gamma_{n}+K_{H} \tilde{H}\right) v+\boldsymbol{Z} \nabla_{\boldsymbol{q}} U=\boldsymbol{Z} \boldsymbol{Y} \tilde{\boldsymbol{\pi}} \\
& \dot{\boldsymbol{s}}_{d}=-\kappa \boldsymbol{s}_{d} \\
& \dot{\boldsymbol{\sigma}}=\operatorname{sgn}(\tilde{\boldsymbol{s}}) \\
& \dot{\boldsymbol{\pi}}=-\boldsymbol{P} \boldsymbol{Y}^{T} \boldsymbol{Z}^{T} \tilde{H} v,
\end{aligned}
$$

where the signals $\boldsymbol{s}_{r}, \dot{\boldsymbol{s}}_{r}$ and $\boldsymbol{s}$ are functions of the state only.

Theorem 1 (Main result): For any $H_{d}>0$, the nonlinear autonomous system (20) has bounded solutions converging to a closed attractive orbit defined by

$$
\begin{gathered}
\mathcal{L}_{d}=\{\chi \mid \tilde{H}=0, \boldsymbol{x}(\boldsymbol{q})=\dot{\boldsymbol{x}}=\mathbf{0}, \\
\left.\boldsymbol{s}_{d}=\mathbf{0}, \boldsymbol{\sigma}=\boldsymbol{\sigma}^{*}, \hat{\boldsymbol{\pi}}=\hat{\boldsymbol{\pi}}^{*}\right\},
\end{gathered}
$$

for some constant $\sigma^{*}, \hat{\pi}^{*}$ satisfying the conditions $\left(\boldsymbol{\Gamma}_{x}+\right.$ $\left.\boldsymbol{K}_{s}\right) \boldsymbol{Q}_{s} \boldsymbol{\sigma}^{*}=\boldsymbol{J}^{+{ }_{M} T} \boldsymbol{Y} \boldsymbol{\pi}$ and $v \boldsymbol{Z Y}\left(\hat{\boldsymbol{\pi}}^{*}-\boldsymbol{\pi}\right)=0$ respectively.

Proof: The proof is based on Theorem 2 that we have formulated in the Appendix.

Using the positive semidefinite $C^{1}$ function of the state (11), it is shown in [19] that $\mathcal{A}=$ $\left\{\chi \mid \boldsymbol{x}(\boldsymbol{q})=\dot{\boldsymbol{x}}=\mathbf{0}, \boldsymbol{s}_{d}=\mathbf{0}, \boldsymbol{\sigma}=\boldsymbol{\sigma}^{*}\right\}$ is the largest invariant set within $E=\left\{\chi \mid \dot{V}_{s}=0\right\}$. In the proof the derivative of $V_{s}$ along the flow of the system is computed, leading to

$$
\dot{V}_{s}=\tilde{\boldsymbol{s}}^{T} \dot{\tilde{\boldsymbol{s}}}=-\tilde{\boldsymbol{s}}^{T} \boldsymbol{Q}_{s} \operatorname{sgn}(\tilde{\boldsymbol{s}})+\tilde{\boldsymbol{s}}^{T} \dot{\boldsymbol{s}}_{r} .
$$

Given Assumption (A2) and choosing $\boldsymbol{K}_{s}$ big enough it is possible to show that $\dot{\boldsymbol{s}}_{r}$ is bounded. If the minimum eigenvalue of $\boldsymbol{Q}_{s}$ is also chosen big enough, then the sliding mode condition $\tilde{\boldsymbol{s}}^{T} \dot{\tilde{\boldsymbol{s}}} \leq-\mu|\tilde{\boldsymbol{s}}|$, with $\mu>0$, is enforced for all time. From that the convergence of $\tilde{s}$ to zero follows and, given its expression, also the convergence of $\boldsymbol{x}(\boldsymbol{q})$ and $\dot{\boldsymbol{x}}$. Therefore, $\mathcal{A}$ will be the largest invariant set within $E$, with $\left(\boldsymbol{\Gamma}_{x}+\boldsymbol{K}_{s}\right) \boldsymbol{Q}_{s} \boldsymbol{\sigma}^{*}=\boldsymbol{J}^{+{ }_{M} T} \boldsymbol{Y} \pi$.

If we prove that $\mathcal{L}_{d}$ is invariant for (20), it is conditionally attractive to $\mathcal{A}$ and the solutions are bounded, then all the requirements of Theorem 2 are satisfied and $\mathcal{L}_{d}$ will turn out to be attractive for the whole system (20). To show the invariance of $\mathcal{L}_{d}$, it is enough to show that it is conditionally invariant to $\mathcal{A}$, since the latter is itself an invariant set. To this end, let us consider the system

$$
\begin{aligned}
& \dot{\boldsymbol{q}}=\boldsymbol{Z}^{T} v \\
& \Lambda_{n} \dot{v}+\left(\Gamma_{n}+K_{H} \tilde{H}\right) v+\boldsymbol{Z} \nabla_{q} U=\boldsymbol{Z} \boldsymbol{Y} \tilde{\boldsymbol{\pi}} \\
& \dot{\boldsymbol{\pi}}=-\boldsymbol{P} \boldsymbol{Y}^{T} \boldsymbol{Z}^{T} \tilde{H} v,
\end{aligned}
$$

with $\boldsymbol{x}(\boldsymbol{q})=\dot{\boldsymbol{x}}=\mathbf{0}$, i.e. (20) conditionally to $\mathcal{A}$. Computing the derivative of $\tilde{H}$ along the flow of (22), results in

$$
\dot{\tilde{H}}=-K_{H} v^{2} \tilde{H}+v \boldsymbol{Z} \boldsymbol{Y} \tilde{\pi},
$$

which, together with (20f), shows that $\tilde{H}$ and $\hat{\pi}^{*}$ will not change when starting from $\mathcal{L}_{d}$. To prove the attractiveness we use the positive definite function

$$
V_{n}=\frac{1}{2}\left(\tilde{H}^{2}+\tilde{\boldsymbol{\pi}}^{T} \boldsymbol{P}^{-1} \tilde{\boldsymbol{\pi}}\right),
$$

with derivative $\dot{V}_{n}=\tilde{H} \dot{\tilde{H}}=-K_{H} v^{2} \tilde{H}^{2} \leq 0$. From LaSalle's invariance theorem, we can conclude that the positive limit set $\mathrm{七}_{n}^{+}$is given by the largest positive invariant set $M_{n}$ within the set $E_{n}=\{\chi \mid v=0$ or $\tilde{H}=0\}$, i.e. the set where $\dot{V}_{n}=0$. Beside $\mathcal{L}_{d}, M_{n}$ contains also equilibrium points satisfying the condition $n\left(\boldsymbol{q}^{*}\right)=\boldsymbol{Z} \boldsymbol{Y} \tilde{\pi}$, so the convergence to $\mathcal{L}_{d}$ cannot be inferred directly. On the other hand, choosing $W_{n}=-V_{n}$ and using Lemma 1 , we can conclude that $E_{n}$ does not contain 


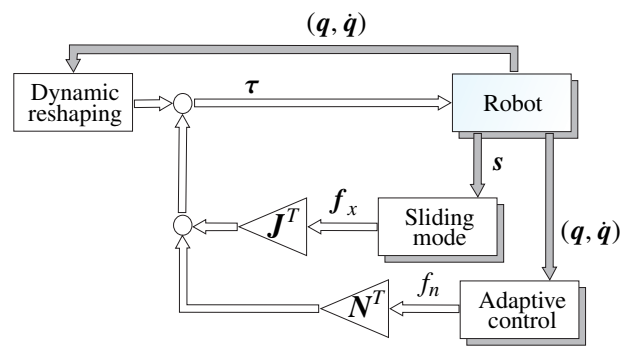

Fig. 4: The proposed controller is given by three subparts.

any stable equilibrium point. Therefore every solution, except for the trivial ones starting from an unstable equilibrium point, approaches $\mathcal{L}_{d}$ as $t \rightarrow \infty$. Boundedness is also always satisfied since we use nonincreasing functions. Finally, $\mathcal{L}_{d}$ is uniquely determined by one parameter, e.g. the value of $n(\boldsymbol{q})$, and therefore it is a closed ${ }^{5}$ attractive orbit in the state space.

\section{Controller discussion}

The control law, as the methods it is based on [19], [20], cannot guarantee asymptotic stability because no stationary value can be known a priori for $\sigma$ and $\hat{\pi}$. These variables are part of the state of the closed loop system together with the original state of the robot. The latter will always converge to a state satisfying the conditions: $\tilde{H}=0$ and $\boldsymbol{x}(\boldsymbol{q})=\dot{\boldsymbol{x}}=\mathbf{0}$, as expressed in Theorem 1. As a result, the physical states of the closed loop system, i.e. the state of the robot, will always converge to the same values.

The torque generated by the controller, as shown in Fig. 4, is due to three contributions: a dynamic reshaping of the system, the torque provided by the sliding mode part of the controller and finally the one generated by the adaptive part. The last two are dynamically decoupled and responsible for forcing the system to evolve on a 1 - dimensional submanifold and for creating the attractive closed orbit respectively. The dynamic reshaping part of the controller compensates for the gravity torque and the coupling terms in the Coriolis matrix. The entry $-\boldsymbol{\Gamma}_{x n}^{T}$ is not actually necessary for the stability analysis, since it has no influence when the system has reached the set $\mathcal{A}$. Nevertheless, in this way, we obtain a power conserving term ${ }^{6}$

$$
\boldsymbol{\tau}_{d}=\boldsymbol{J}_{N}^{T}\left[\begin{array}{cc}
\mathbf{0} & \boldsymbol{\Gamma}_{x n} \\
-\boldsymbol{\Gamma}_{x n}^{T} & 0
\end{array}\right]\left[\begin{array}{c}
\dot{\boldsymbol{x}} \\
v
\end{array}\right],
$$

where $\tau_{d}^{T} \dot{\boldsymbol{q}}=0$. Although $\tau_{d}$ compensates for the coupling terms in the Coriolis matrix, it does not decouple the dynamics in the two spaces. The remaining blocks of the Coriolis matrix are, in fact, still function of the whole state.

In the analysis it is required that $\boldsymbol{x}(\boldsymbol{q})$ and $\dot{\boldsymbol{x}}$ converge to zero. Therefore, a chattering free sliding mode approach is a perfect candidate for the task. On the other hand, a sliding mode control cannot be used for the zero dynamics.

\footnotetext{
${ }^{5}$ It can be seen as the level set of a positive definite and radially unbounded function.

${ }^{6}$ This is a generalization of the nominal control in [6], [26].
}

Although the energy error can be used as sliding variable with relative degree $r=1$, after differentiation the control input would not appear multiplied by the sliding variable itself, as it is for example in [20]. An adaptive method can be used in this case. It is also clear that, once it has been chosen to adapt the parameters in such a way that convergence is guaranteed for the zero dynamics, the same parameters cannot be adapted with a different law to guarantee the convergence to the constraint submanifold. In other word, it is possible to design the adaptation law for one of the two subparts of the controller. This explains why we could not use an adaptive approach also to enforce the constraints.

Finally, in our framework we require a perfect knowledge of the system matrices. The main reason why this cannot be avoided in the current state is that we need to compute the nullspace projector $N(q)$, for which the inertia matrix of the robot is required.

\section{EXPERIMENT}

The proposed control law is evaluated in an experiment with the humanoid robot TORO described in [27], which was developed at the German Aerospace Center (DLR). The robot has 27 degrees of freedom with a height of $1.7 \mathrm{~m}$ and a mass of about $77.5 \mathrm{~kg}$.

In [5] a detailed analysis for different type of constraints and different choices of the virtual potential function $U(\boldsymbol{q})$ was presented. Therefore in this section we will only focus on comparing the proposed control law to the previous approach for given functions $\boldsymbol{x}(\boldsymbol{q})$ and $U(\boldsymbol{q})$.

a) Friction model: We assume that the unknown torques $\tau_{f}$ in (1) can be expressed as

$$
\boldsymbol{\tau}_{f}=\operatorname{diag}\left(\boldsymbol{f}_{v}\right) \dot{\boldsymbol{q}}+\operatorname{diag}\left(\boldsymbol{f}_{s}\right) \operatorname{sgn}(\dot{\boldsymbol{q}}),
$$

where $\boldsymbol{f}_{v}, \boldsymbol{f}_{s} \in \mathbb{R}^{n}$ are the vectors of the unknown coefficients of the viscous and Coulomb friction respectively. The previous torques can be expressed using

$$
\boldsymbol{Y}=[\operatorname{diag}(\dot{\boldsymbol{q}}) \quad \operatorname{diag}(\operatorname{sgn}(\dot{\boldsymbol{q}}))], \quad \boldsymbol{\pi}=\left[\begin{array}{ll}
\boldsymbol{f}_{v}^{T} & \boldsymbol{f}_{s}^{T}
\end{array}\right]^{T} .
$$

b) Desired configuration and constraint function: In this example the constraint submanifold is given directly in the joint space. TORO is using its legs to maintain balance, while the 12 joints of the arms are forced on a 1 dimensional submanifold defined by the constraint function

$$
x_{i}(\boldsymbol{q})=q_{i}-q_{d_{i}}-q_{c_{i}} \quad i=2, \ldots, 12,
$$

where $\boldsymbol{q}_{c}$ is used to couple the elbow joint of the right arm to the second joint of the shoulder of the same arm, i.e. $q_{c_{4}}=5 q_{1}$ while the rest of the entries are zero. Finally $\boldsymbol{q}_{d}$ is chosen to be the desired configuration shown in Fig. 5. It is worth to notice that choosing $q_{d_{1}}=0$, then the condition $\boldsymbol{x}\left(\boldsymbol{q}_{d}\right)=\mathbf{0}$ is satisfied.

c) Potential function: A simple choice for the virtual potential is given by

$$
U(\boldsymbol{q})=\frac{1}{2} k_{n}\left\|\boldsymbol{q}-\boldsymbol{q}_{d}\right\|^{2},
$$




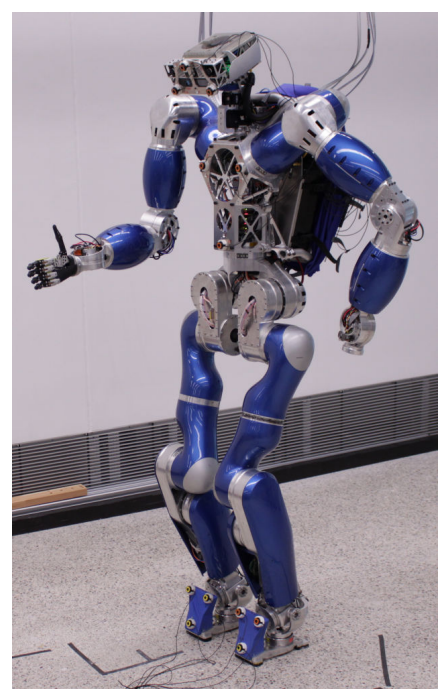

Fig. 5: Desired configuration.

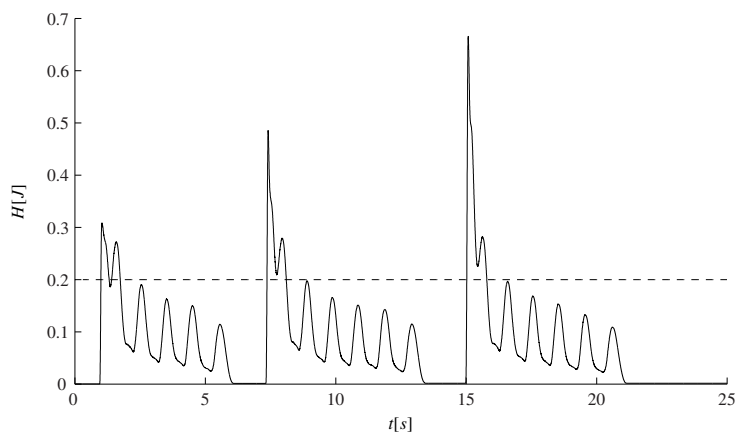

Fig. 6: Energy function (continuous line) and desired value (dashed line) without friction compensation obtained in the experiment.

where $k_{n}=40 \mathrm{Nm} /$ rad. $U(\boldsymbol{q})$ so defined is clearly positive definite on the 1 - dimensional submanifold and with its minimum at $\boldsymbol{q}_{d}$.

d) Results: Given the definition of $\boldsymbol{x}(\boldsymbol{q})$, the resulting motion will be an oscillation similar to a handshake in which the first shoulder joint works as limit cycle generator with the elbow joint coupled to it, while the remaining ones will keep the desired position.

In order to highlight the role of the friction compensation the gain $K_{H}$ is set very low $\left(K_{H}=5 \mathrm{~s} / \mathrm{rad}^{2}\right)$. Because of that the convergence of the energy error is poor and, in case of no compensation, the friction is the predominant effect and no oscillation is achieved, although the robot is pushed three times by the experimenter. The plot of the energy is shown in Fig. 6, where the three high peaks correspond to the three interactions between the robot and the experimenter. On the other hand, using the friction compensation, the energy is oscillating around the desired value after few seconds, as shown in Fig. 7. In both cases the value of the desired energy is $H_{d}=0.2 \mathrm{~J}$.

The values of the gains used for the experiment are listed in Table I.

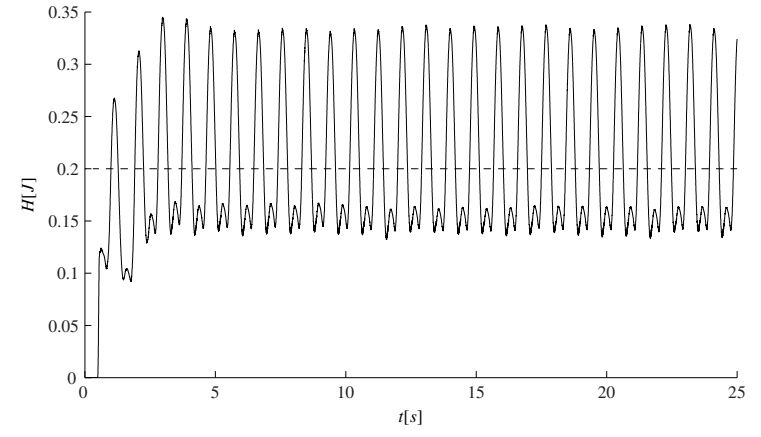

Fig. 7: Energy function (continuous line) and desired value (dashed line) with friction compensation obtained in the experiment.

e) Discussion: In Fig. 7 the energy oscillates around its desired value, although convergence was theoretically expected. Measurement noise, flexibility of the structure and model uncertainties are a possible cause for this phenomenon. We suppose that the mismatch between the real model and the one used by the controller is the main reason for the oscillations in the recorded signal. The behavior can, in fact, be reproduced in simulation when using an incorrect model for the controller and disappears when the model is perfectly known, as it can be seen in Fig. 8. In the simulation the robot starts with a higher deviation from the submanifold compared to the experiment. This causes the big overshoot $^{7}$ in the plot, since the total kinetic energy is used in the definition of $H(\boldsymbol{q}, \dot{\boldsymbol{q}})$, but still a perfect convergence is achieved. The friction model used is as in (26), where the values of the parameters are unknown for the same controller used for the experimental setup. This kind of model uncertainty, as discussed in Section III-D, cannot be handled by the current state of the controller. Nevertheless, the experiment gives the opportunity to test the robustness of the proposed approach. Although it was not formally shown, the behavior results to be periodic even if the energy error does not converge exactly to zero, showing that the effect of the model uncertainty is a distortion of the expected attractive closed orbit.

\section{CONCLUSIONS}

We have addressed the problem of generating attractive closed orbits, for fully actuated robots. In order to cope with friction always present in real systems, we have extended our previous results using a combination of standard approaches

${ }^{7}$ During this phase the term proportional to the energy error is acting like a damping, so no unexpected oscillations will be produced.

TABLE I: Gains used for one arm in the experiment.

\begin{tabular}{cll} 
Parameter & Value & Unit \\
\hline $\boldsymbol{K}$ & 30 & $1 / \mathrm{s}$ \\
$\boldsymbol{Q}_{s}$ & 0.07 & $1 / \mathrm{s}$ \\
$\boldsymbol{D}_{S}$ & $\operatorname{diag}(32,60,50,66,51,51)$ & $1 / \mathrm{s}$ \\
$\boldsymbol{K}_{s}$ & $\operatorname{diag}(5,2.5,2,1.5,1,1)$ & $\mathrm{Nms} / \mathrm{rad}^{2}$ \\
$\boldsymbol{P}$ & $\operatorname{diag}(10, \ldots, 10,1, \ldots, 1)$ & $\mathrm{rad}^{2} / \mathrm{Nms}^{2}$
\end{tabular}




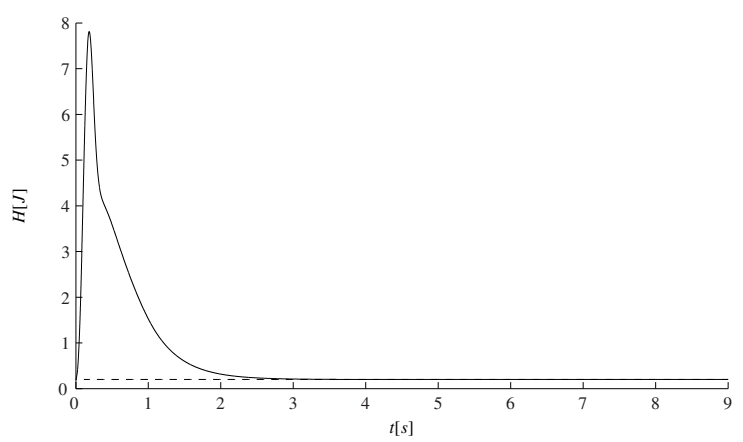

Fig. 8: Energy function (continuous line) and desired value (dashed line) with friction compensation obtained in simulation.

for uncertain models. Standard friction compensation techniques cannot be applied directly if we want to keep the design of the control law separated in two steps, as in our previous work. The reason is that no interferences between the two parts of the controller are allowed when compensating the friction. The approach has been validated in an experiment using the humanoid robot TORO, which confirms the improvement in the performances compared to our previous approach.

A possible scenario where to apply these concepts is bipedal robotics, where often the goal is to obtain periodic motion patterns. The hybrid nature of such systems sets some challenges that need to be overcome before using directly the results of this paper. Nevertheless, assuming a simplified switching behavior of the dynamic equations and step to step control strategies could allow to use a similar approach for the contact phase of the robot. Such a topic is currently under investigation. In particular, the use of elastic actuators appears very appealing in these cases. In the last years these actuators are spreading more and more, because of the possibility to achieve higher performances and improve the efficiency of actuation through the energy stored in the springs. Therefore, using an energy based approach, we expect to be able to exploit the benefits of such actuators, for example replacing the virtual potential used in this work with the real potential of the elastic elements.

\section{APPENDIX}

Let us consider the first order dynamic system

$$
\dot{\chi}=f(\chi),
$$

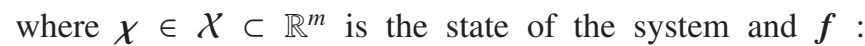
$X \rightarrow \mathbb{R}^{m}$ is a Lebesgue measurable function. The equation is understood in the sense of Filippov [28], i.e. (29) is replaced by the equivalent differential inclusion obtained using its convex regularization [28]. Existence of solutions can be guaranteed with the notion of upper-continuity of set-valued functions [28]. We denote with $\chi\left(t ; \chi_{0}\right)$ the absolutely continuous solution satisfying the differential inclusion almost everywhere, starting from $\chi_{0}$ and evaluated at the time instant $t$, with $\chi\left(0 ; \chi_{0}\right)=\chi_{0}$.

Given a set $\Omega \subset \mathcal{X}$, the following definitions apply. a) (Distance): $d(\chi, \Omega) \triangleq \min _{y \in \Omega}\|\chi-y\|$

b) (Open ball): $B_{\epsilon}(\Omega) \triangleq\{\chi \in \mathcal{X} \mid d(\chi, \Omega)<\epsilon\}$

c) (Closed ball): $\bar{B}_{\epsilon}(\Omega) \triangleq\{\chi \in \mathcal{X} \mid d(\chi, \Omega) \leq \epsilon\}$

d) (Sphere): $S_{\epsilon}(\Omega) \triangleq\{\chi \in \mathcal{X} \mid d(\chi, \Omega)=\epsilon\}$

e) (Conditional attractiveness): $\Omega$ is attractive conditionally to $\mathcal{A}$ if $\Omega \subset \mathcal{A}$ and $\exists \delta>0$ such that $\forall \chi_{0} \in$ $B_{\delta}(\Omega) \cap \mathcal{A} \Rightarrow \lim _{t \rightarrow \infty} d\left(\chi\left(t ; \chi_{0}\right), \Omega\right)=0$

Lemma 1 ( [29]): Let $€^{+}$be the positive limit set $(\omega-$ limit set) of a bounded solution of a dynamical system. Given some technical assumptions [29], a $C^{1}$ scalar function of the state $W$ such that $\dot{W} \geq 0$ along the flow of the system and indicating with $E$ the set where $\dot{W}=0$, then $\mathrm{七}^{+}$and $E$ have nonempty intersection. Moreover if $\iota^{+}$does not reduce to a single point, then $\mathrm{七}^{+} \cap E$ does not contain any stable equilibrium point.

Lemma 2 ( [30]): If a solution $\chi\left(t ; \chi_{0}\right)$ of (29) is bounded and belongs to $X$ for $t \geq 0$, then its positive limit set $\mathrm{七}^{+}$ is a nonempty, compact, invariant set. Moreover, $\chi\left(t ; \chi_{0}\right)$ approaches $\mathrm{E}^{+}$as $t \rightarrow \infty$.

Theorem 2 (Convergence with semidefinite functions): Let $\Omega$ be an invariant set for (29), and let $V(\chi)$ be a differentiable function defined in $B_{v}(\Omega) \subset \mathcal{X}$ such that $V(\chi) \geq 0 \forall \chi \in B_{v}(\Omega), V(\Omega)=0$ and $\dot{V}(\chi) \leq 0 \forall \chi \in B_{v}(\Omega)$. If $\Omega$ is attractive conditionally to the largest positively invariant set $\mathcal{M}$ within $E=\left\{\chi \in B_{v}(\Omega) \mid \dot{V}(\chi)=0\right\}$ and the solutions are bounded, then $\Omega$ is attractive.

Proof: Let $\alpha=\min _{\chi \in S_{\nu}(\Omega)} V(\chi), \alpha>0$, and take $\beta$ such that $0<\beta<\alpha, \Omega_{\beta}=\left\{\chi \in B_{v}(\Omega) \mid V(\chi) \leq \beta\right\}$. Since $\Omega_{\beta}$ is a compact positively invariant set, by virtue of LaSalle's invariance theorem then every solution starting in $\Omega_{\beta}$ approaches $\mathcal{M}$ as $t \rightarrow \infty$. Since the solutions are bounded, then $\exists \delta=\delta\left(\chi_{0}\right)>0$ such that $\chi\left(t ; \chi_{0}\right) \in B_{\delta}(\Omega), \forall t \geq 0$. Using Lemma 2, the positive limit set $\mathrm{七}^{+}$is such that $\mathfrak{七}^{+} \in$ $B_{\delta}(\Omega) \cap \mathcal{M}$. Additionally, since $\Omega$ is attractive conditionally to $\mathcal{M}$, then $\lim _{t \rightarrow \infty} d\left(\chi\left(t ; \chi_{0}\right), \Omega\right)=0$ if $\chi_{0} \in B_{\delta}(\Omega) \cap \mathcal{M}$. Now let us assume by contradiction that $\mathrm{E}^{+}$is not $\Omega$. Choosing $\chi_{0}=\boldsymbol{y} \in \mathrm{七}^{+} \neq \Omega$ we reach a contradiction.

\section{ACKNOWLEDGMENT}

This research is partly supported by the Initiative and Networking Fund of the Helmholtz Association through a Helmholtz Young Investigators Group (Grant no. VH-NG808).

\section{REFERENCES}

[1] T. McGeer, "Passive dynamic walking," Int. Journal of Robotics Research, vol. 9, pp. 62-82, 1990.

[2] H. Geyer, A. Seyfarth, and R. Blickhan, "Compliant leg behavior explains basic dynamics of walking and running," Proceedings of the Royal Society B, vol. 273, p. 28612867, Nov. 2006.

[3] G. Garofalo, C. Ott, and A. Albu-Schäffer, "Walking control of fully actuated robots based on the bipedal slip model," in IEEE Int. Conf. on Robotics and Automation (ICRA), Saint Paul, USA, May 2012, pp. 1999-2004.

[4] E. R. Westervelt, J. W. Grizzle, C. Chevallereau, J. H. Choi, and B. Morris, Feedback Control of Dynamic Bipedal Robot Locomotion. CRC Press, 2007.

[5] G. Garofalo, C. Ott, and A. Albu-Schäffer, "Orbital stabilization of mechanical systems through semidefinite Lyapunov functions," in American Control Conference (ACC), Washington DC, USA, June 2013, pp. $5735-5741$. 
[6] V. Duindam and S. Stramigioli, "Passive asymptotic curve tracking," in Proceedings of the IFAC Workshop on Lagrangian and Hamiltonian Methods for Nonlinear Control, Seville, Spain, Apr. 2003, pp. 229234.

[7] M. Taniguchi and K. Fujimoto, "Asymptotic path following and velocity control of port-Hamiltonian systems," in European Control Conference (ECC), Budapest, Hungary, Aug. 2009, pp. $236-241$.

[8] C. Canudas-de-Wit, B. Espiau, and C. Urrea, "Orbital stabilization of underactuated mechanical systems," in Triennial World Congress of the International Federation of Automatic Control, Barcelona, Spain, July 2002, pp. 893-898.

[9] J. Park, W. Chung, and Y. Youm, "On dynamical decoupling of kinematically redundant manipulators," in IEEE/RSJ International Conference on Intelligent Robots and Systems, 1999, pp. $1495-1500$.

[10] C. Ott, Cartesian Impedance Control of Redundant and Flexible-Joint Robots, ser. Springer Tracts in Advanced Robotics. Berlin: SpringerVerlag, 2008.

[11] C. Ott, A. Kugi, and Y. Nakamura, "Resolving the problem of non-integrability of nullspace velocities for compliance control of redundant manipulators by using semi-definite Lyapunov functions," in IEEE Int. Conf. on Robotics and Automation (ICRA), Pasadena, USA, May 2008, pp. 1456-1463.

[12] D. Lakatos, G. Garofalo, F. Petit, C. Ott, and A. Albu-Schäffer, "Modal limit cycle control for variable stiffness actuated robots," in IEEE Int. Conf. on Robotics and Automation (ICRA), Karlsruhe, Germany, May 2013, pp. 4934-4941.

[13] T. Petric, L. Zlajpah, G. Garofalo, and C. Ott, "Walking control using adaptive oscillators combined with dynamic movement primitives," in 22nd International Workshop on Robotics in Alpe-Adria-Danube Region (RAAD), PortoroÅ, Slovenia, Sept. 2013, pp. 204-211.

[14] D. Lakatos, G. Garofalo, A. Dietrich, and A. Albu-Schäffer, "Jumping control for compliantly actuated multilegged robots," in IEEE Int. Conf. on Robotics and Automation (ICRA), Hong Kong, China, May 2014, pp. 4562-4568.

[15] G. Garofalo, J. Englsberger, and C. Ott, "On the regulation of the energy of elastic joint robots: excitation and damping of oscillations," in American Control Conference (ACC), Chicago, USA, July 2015, pp. 4825-4831.

[16] S. M. Shahruz, G. Langari, and M. Tomizuka, "Design of robust PDtype control laws for robotic manipulators with parametric uncertainties," in American Control Conference (ACC), Chicago, USA, June 1992, pp. 2967-2968.
[17] C.-L. Chen and R.-L. Xu, "Tracking control of robot manipulator using sliding mode controller with performance robustness," ASME Journal of Dynamic Systems, Measurement, and Control, vol. 121, no. 1, pp. 64-70, 1999.

[18] A. A. Pervozvanski and L. B. Freidovich, "Robust stabilization of robotic manipulators by PID controllers," Dynamics and Control, vol. 9, no. 3, pp. 203-222, 1999

[19] V. Parra-Vega, S. Arimoto, Y.-H. Liu, G. Hirzinger, and P. Akella, "Dynamic sliding PID control for tracking of robot manipulators: theory and experiments," IEEE Trans. Robotics and Automation, vol. 19, no. 6, pp. 967-976, 2003.

[20] J.-J. E. Slotine and W. Li, "On the adaptive control of robot manipulators," Int. Journal of Robotics Research, vol. 6, pp. 49-59, 1987.

[21] R. Ortega and M. W. Spong, "Adaptive motion control of rigid robots: a tutorial," in IEEE Conf. on Decision and Control, Texas, USA, Dec. 1988 , pp. $1575-1584$.

[22] N. Saddegh and R. Horowitz, "Stability and robustness analysis of a class of adaptive controllers for robotic manipulators," Int. Journal of Robotics Research, vol. 9, no. 3, pp. 74-92, 1990.

[23] T. Frankel, The Geometry of Physics: An Introduction. New York: Cambridge University Press, 2004.

[24] V. Utkin, Sliding modes in control and optimization. New York: Springer-Verlag, 1992.

[25] J. Baillieul, "Kinematic programming alternatives for redundant manipulators," in IEEE Int. Conf. on Robotics and Automation (ICRA), St. Louis, USA, Mar. 1985, pp. 722-728.

[26] V. Duindam and S. Stramigioli, "Port-based asymptotic curve tracking for mechanical systems," European Journal of Control, vol. 10, pp. 411-420, 2004.

[27] J. Englsberger, A. Werner, C. Ott, B. Henze, M. A. Roa, G. Garofalo, R. Burger, A. Beyer, O. Eiberger, K. Schmid, and A. AlbuSchäffer "Overview of the torque-controlled humanoid robot TORO," in IEEE/RAS Int. Conf. on Humanoid Robots, Madrid, Spain, Nov. 2014, pp. 916-923.

[28] A. F. Filippov, Differential equations with discontinuous righthand sides, ser. Mathematics and its applications. Dordrecht: Kluwer Academic, 1988

[29] A. Arsie and C. Ebenbauer, "Refining LaSalle's invariance principle," in American Control Conference (ACC), Saint Louis, USA, June 2009, pp. $108-112$.

[30] H. K. Khalil, Nonlinear Systems. New Jersey: Prentice Hall, 2002. 